\title{
A Versatile Set of Aminooxy Amino Acids for the Synthesis of Neoglycopeptides
}

\author{
Michael R. Carrasco* and Ryan T. Brown
}

Table of contents

$\begin{array}{ll}\text { General experimental procedures } & \text { S2 }\end{array}$

Figure S1. ${ }^{1} \mathrm{H}$ NMR spectrum of $\mathbf{1 b} \quad$ S3

Figure S2. ${ }^{13} \mathrm{C}$ NMR spectrum of $\mathbf{1 b} \quad$ S4

Figure S3. ${ }^{1} \mathrm{H}$ NMR spectrum of $\mathbf{2 b} \quad$ S5

Figure S4. ${ }^{13} \mathbf{C}$ NMR spectrum of $\mathbf{2 b} \quad$ S6

Figure S5. ${ }^{1} \mathrm{H}$ NMR spectrum of $\mathbf{3 b} \quad$ S7

Figure S6. ${ }^{13} \mathrm{C}$ NMR spectrum of $\mathbf{3 b} \quad$ S8

Figure S7. ${ }^{1} \mathrm{H}$ NMR spectrum of $\mathbf{4 b} \quad$ S9

Figure S8. ${ }^{13} \mathrm{C}$ NMR spectrum of $\mathbf{4 b} \quad \mathbf{b} 10$

Figure S9. ${ }^{1} \mathrm{H}$ NMR spectrum of $5 \quad$ S11

Figure S10. ${ }^{13} \mathrm{C}$ NMR spectrum of $5 \quad \mathrm{~S} 12$

Figure S11. ${ }^{1} \mathrm{H}$ NMR spectrum of $6 \quad$ S13

Figure S12. ${ }^{13} \mathrm{C}$ NMR spectrum of $6 \quad \mathrm{~S} 14$

Figure S13. ${ }^{1} \mathrm{H}$ NMR spectrum of $7 \quad \mathrm{~S} 15$

Figure S14. ${ }^{13} \mathrm{C}$ NMR spectrum of $7 \quad$ S16

$\begin{array}{ll}\text { Figure S15. }{ }^{1} \mathrm{H} \text { NMR spectrum of } 8 & \text { S17 }\end{array}$

Figure S16. ${ }^{13} \mathrm{C}$ NMR spectrum of $8 \quad$ S18

$\begin{array}{ll}\text { Figure S17. }{ }^{1} \mathrm{H} \text { NMR spectrum of } 9 & \text { S19 }\end{array}$

Figure S18. ${ }^{13} \mathrm{C}$ NMR spectrum of $9 \quad$ S20

Figure S19. HPLC chromatograms of $\mathbf{1 4}$ and $\mathbf{1 8} \quad$ S21

Figure S20. HPLC chromatograms of $\mathbf{1 5}$ and $\mathbf{1 9} \quad$ S22

Figure S21. HPLC chromatograms of $\mathbf{1 6}$ and $\mathbf{2 0} \quad$ S23

Figure S22. HPLC chromatogram of $\mathbf{1 7} \quad$ S24 


\section{General experimental procedures.}

Unless otherwise noted, all reactions were run at $\mathrm{rt}$ and under ambient atmosphere. Chromatographic separations were performed using silica gel (230-400 mesh). Organic solutions were dried with $\mathrm{Na}_{2} \mathrm{SO}_{4}$, and solvents were removed using standard rotary evaporation under reduced pressure. Products were dried under high vacuum. Commercial reagents were used in all cases without further purification. Spectral characterizations of $\mathbf{1 b}, \mathbf{2} \mathbf{b}$, and $\mathbf{3 b}$ were performed at elevated temperatures because of the presence of distinct rotational isomers under most conditions.

SPPS was performed using commercial MBHA resin using standard Boc-chemistry-based procedure ${ }^{10}$ with the following variations. The amino acids were activated with 2-(1H-benzotriazole-1-yl)1,1,3,3-tetramethyluronium hexafluorophosphate (HBTU) and $\mathrm{NEt}(i-\mathrm{Pr})_{2}$ in DMF. All commercial amino acids were used in a 10-fold excess to the resin loading and allowed to react for $10 \mathrm{~min}$. $\mathbf{1 b}, \mathbf{2 b}, \mathbf{3 b}$ and $\mathbf{4 b}$ were used in a 2 to 3 -fold excess and allowed to react for $25 \mathrm{~min}$. Boc deprotections were carried out using neat TFA. Resin washings between steps were performed with a continuous flow of DMF for 1 min. Final deprotection and resin cleavage was accomplished by treatment with liquid HF or bromotrimethylsilane:TFA:thioanisole (v:v:v, 0.6:3:0.8). 
Figure S1. ${ }^{1} \mathrm{H}$ NMR spectrum of $\mathbf{1 b}\left(400 \mathrm{MHz}, \mathrm{CDCl}_{3}, 55^{\circ} \mathrm{C}\right)$.

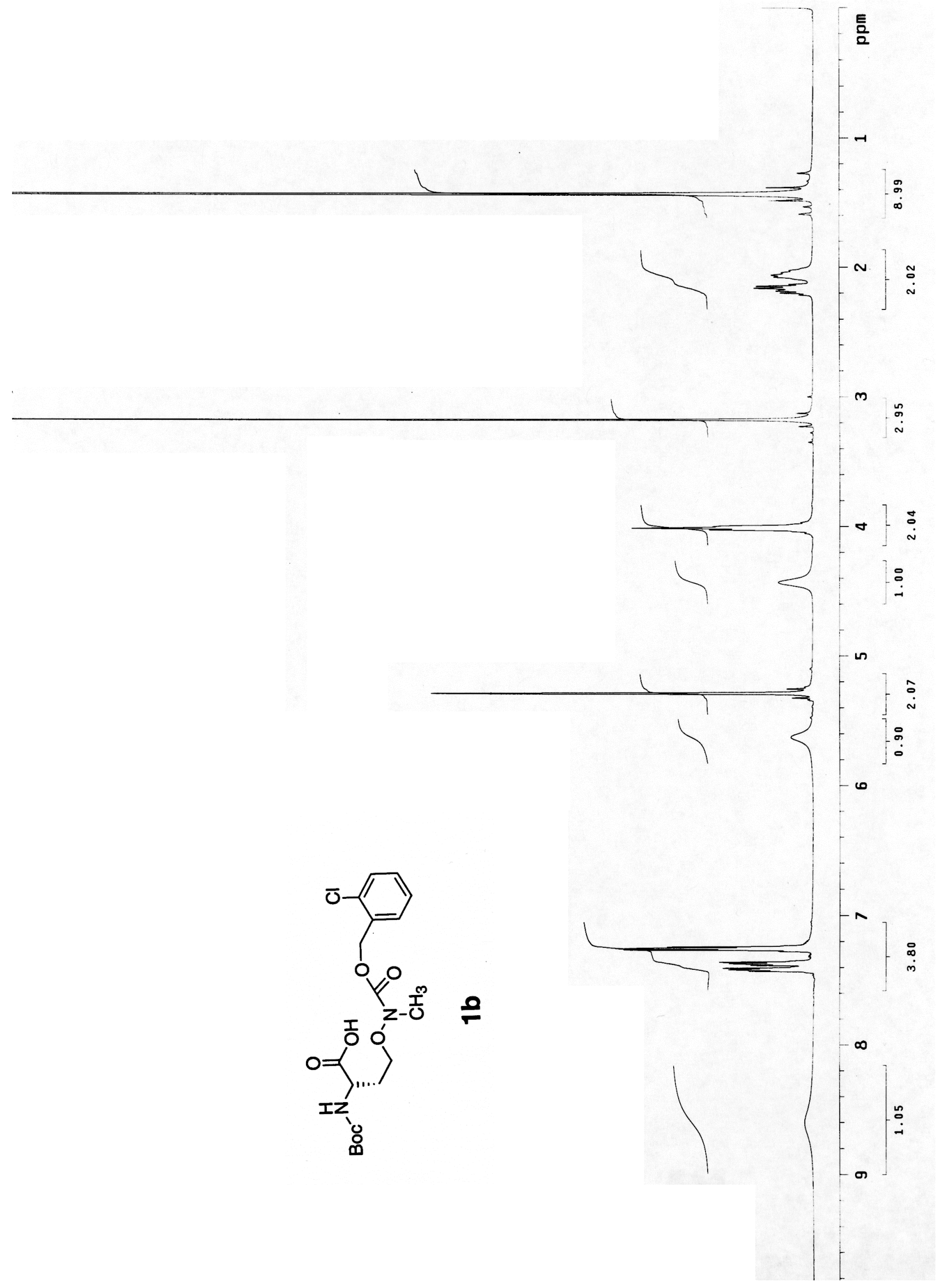


Figure S2. ${ }^{13} \mathrm{C}$ NMR spectrum of $\mathbf{1 b}\left(100 \mathrm{MHz}, \mathrm{CDCl}_{3}, 55^{\circ} \mathrm{C}\right)$.

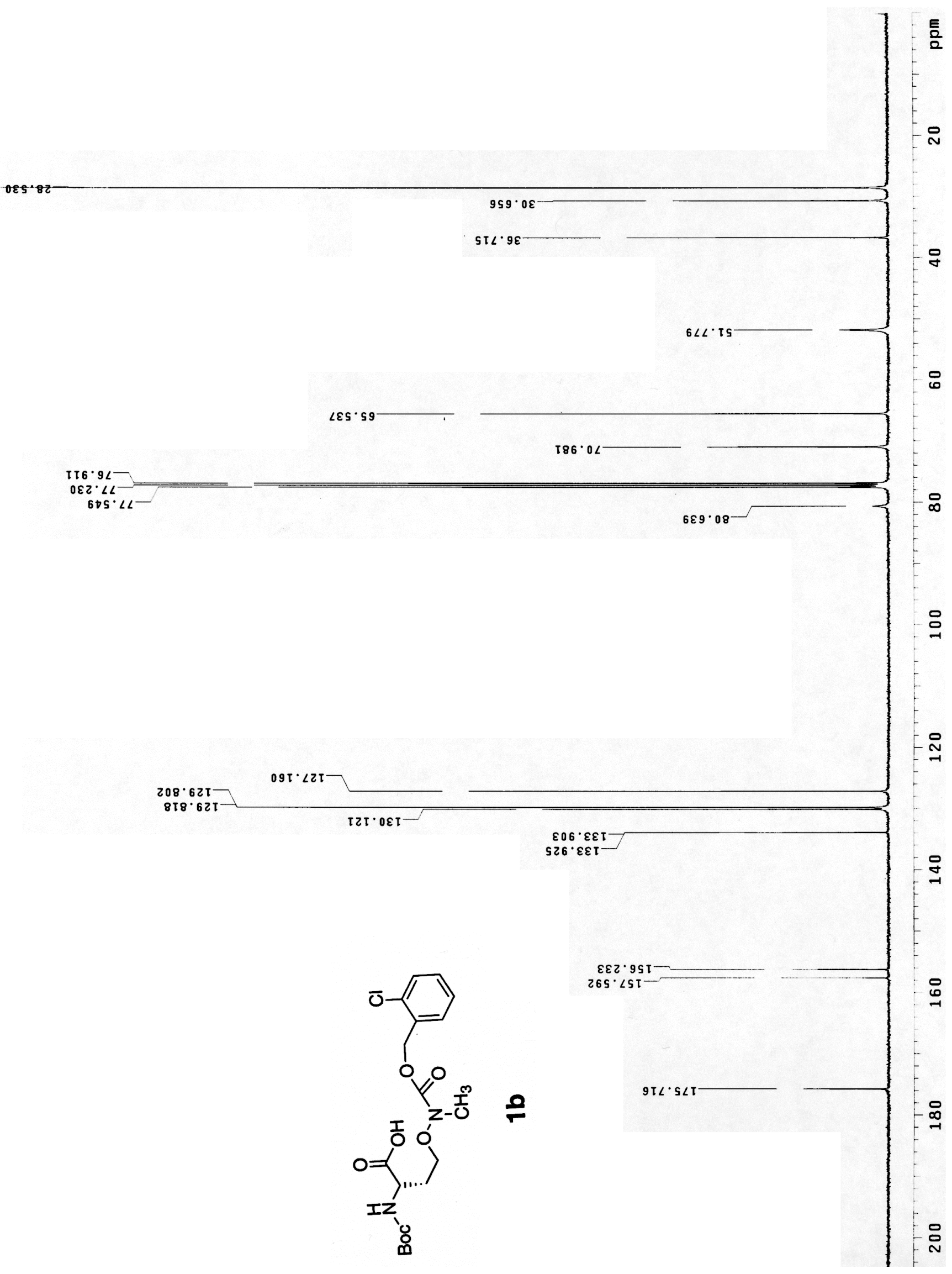


Figure S3. ${ }^{1} \mathrm{H}$ NMR spectrum of $\mathbf{2 b}\left(400 \mathrm{MHz}, \mathrm{CD}_{3} \mathrm{CN}, 55^{\circ} \mathrm{C}\right)$.

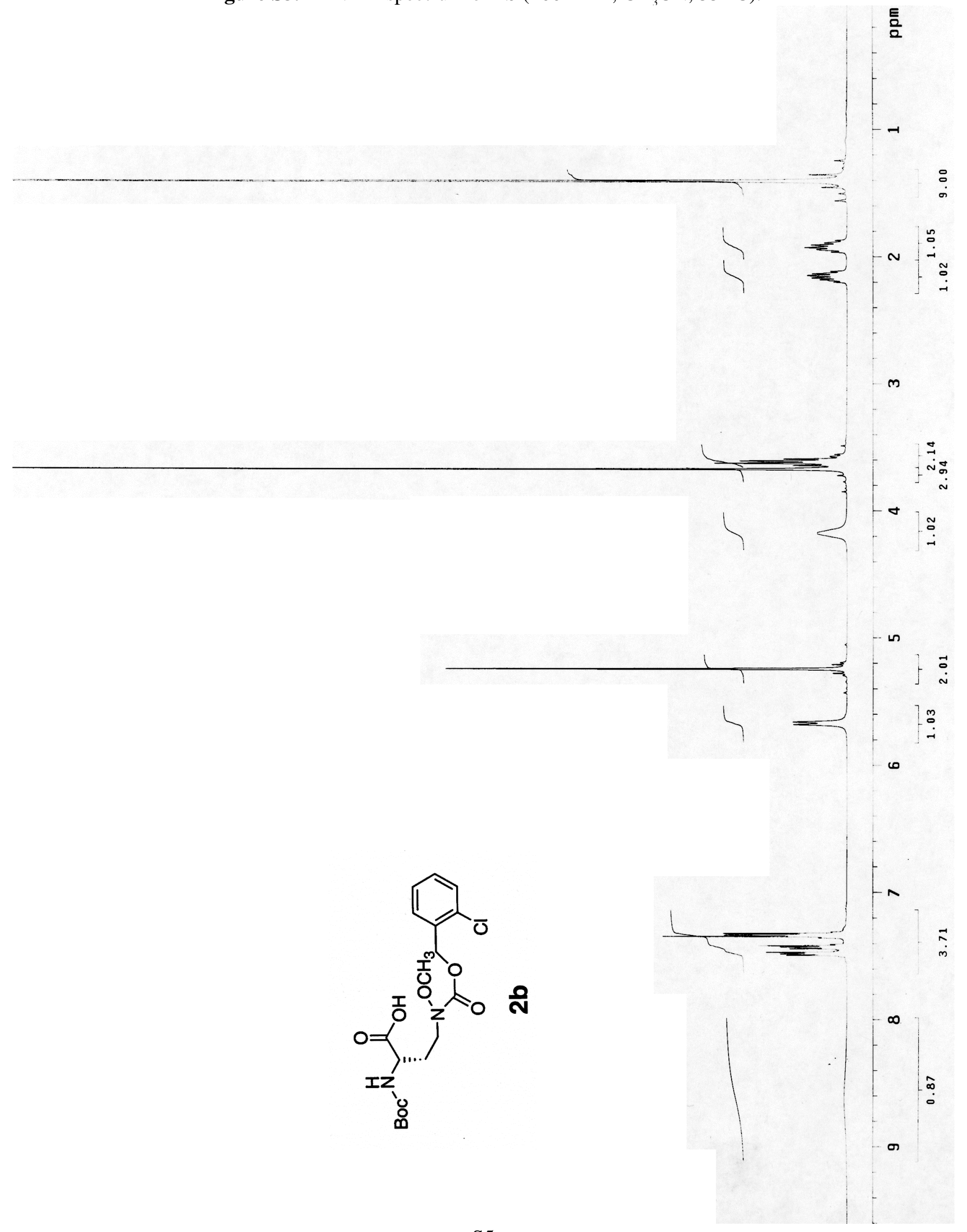


Figure S4. ${ }^{13} \mathrm{C}$ NMR spectrum of $\mathbf{2 b}\left(100 \mathrm{MHz}, \mathrm{CD}_{3} \mathrm{CN}, 55^{\circ} \mathrm{C}\right)$.

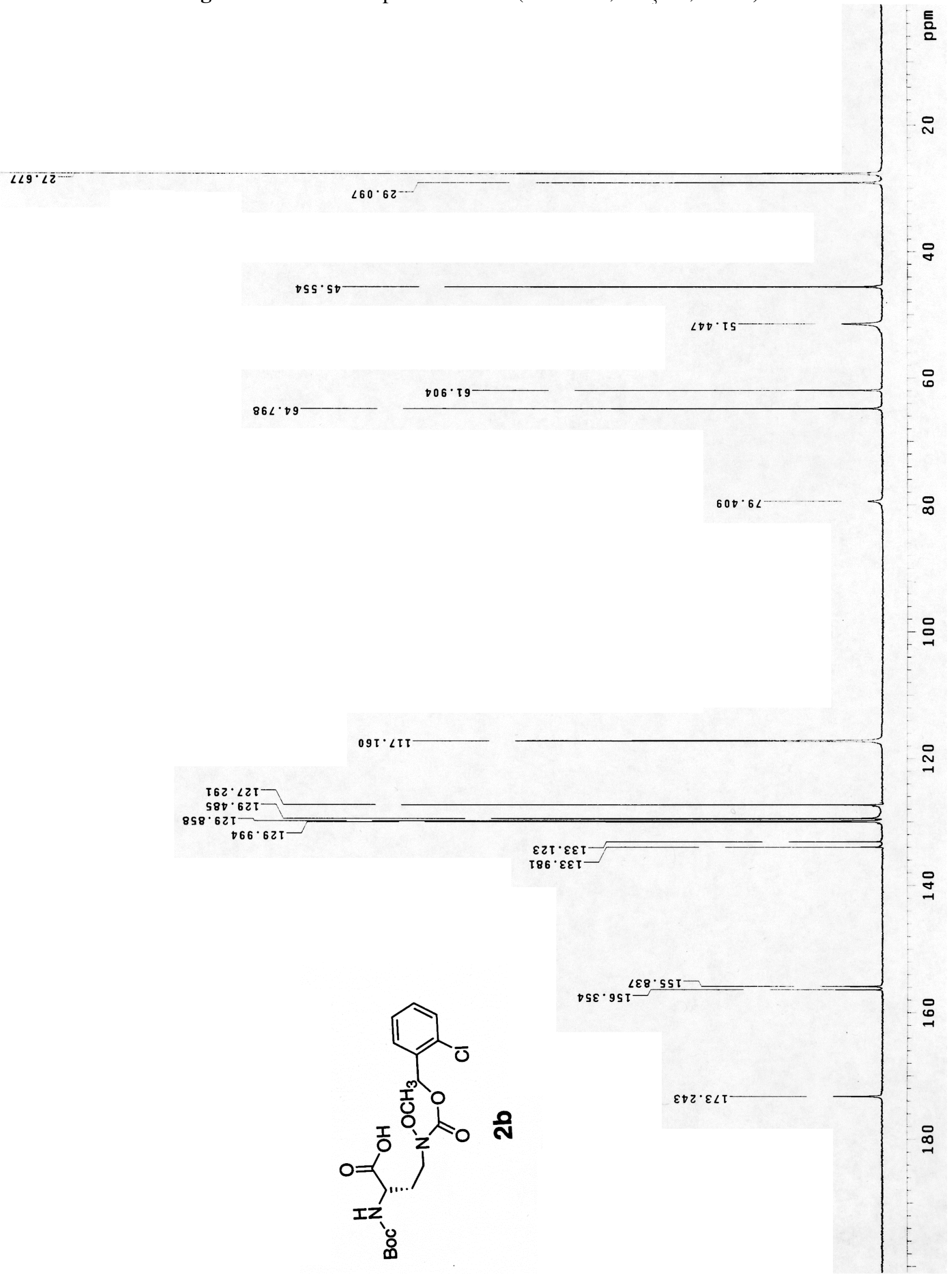


Figure S5. ${ }^{1} \mathrm{H}$ NMR spectrum of $\mathbf{3 b}\left(400 \mathrm{MHz}, \mathrm{CD}_{3} \mathrm{CN}, 55^{\circ} \mathrm{C}\right)$.

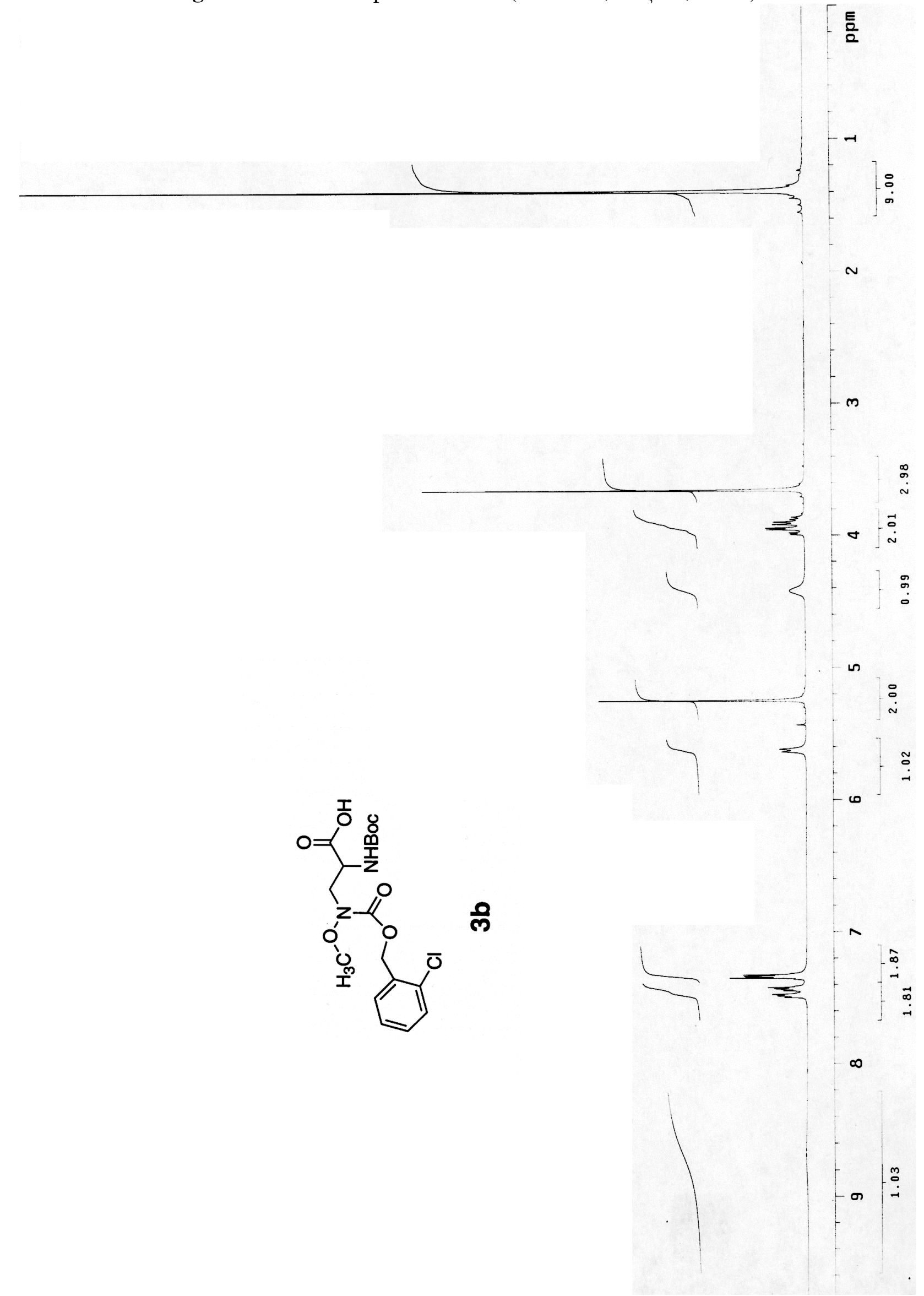


Figure S6. ${ }^{13} \mathrm{C}$ NMR spectrum of $\mathbf{3 b}\left(100 \mathrm{MHz}, \mathrm{CD}_{3} \mathrm{CN}, 55^{\circ} \mathrm{C}\right)$.

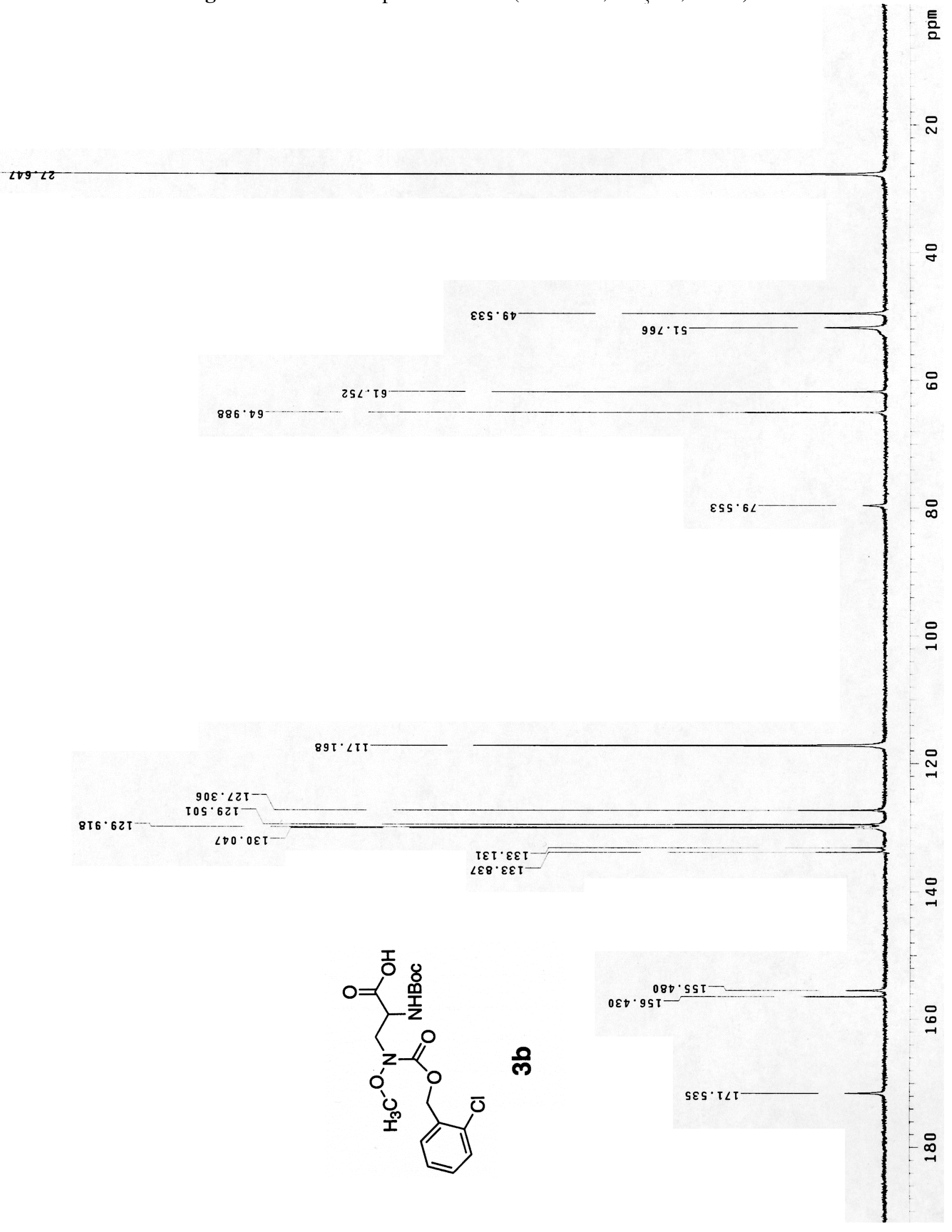


Figure S7. ${ }^{1} \mathrm{H}$ NMR spectrum of $\mathbf{4 b}\left(400 \mathrm{MHz}, \mathrm{CD}_{3} \mathrm{CN}, 55^{\circ} \mathrm{C}\right)$.

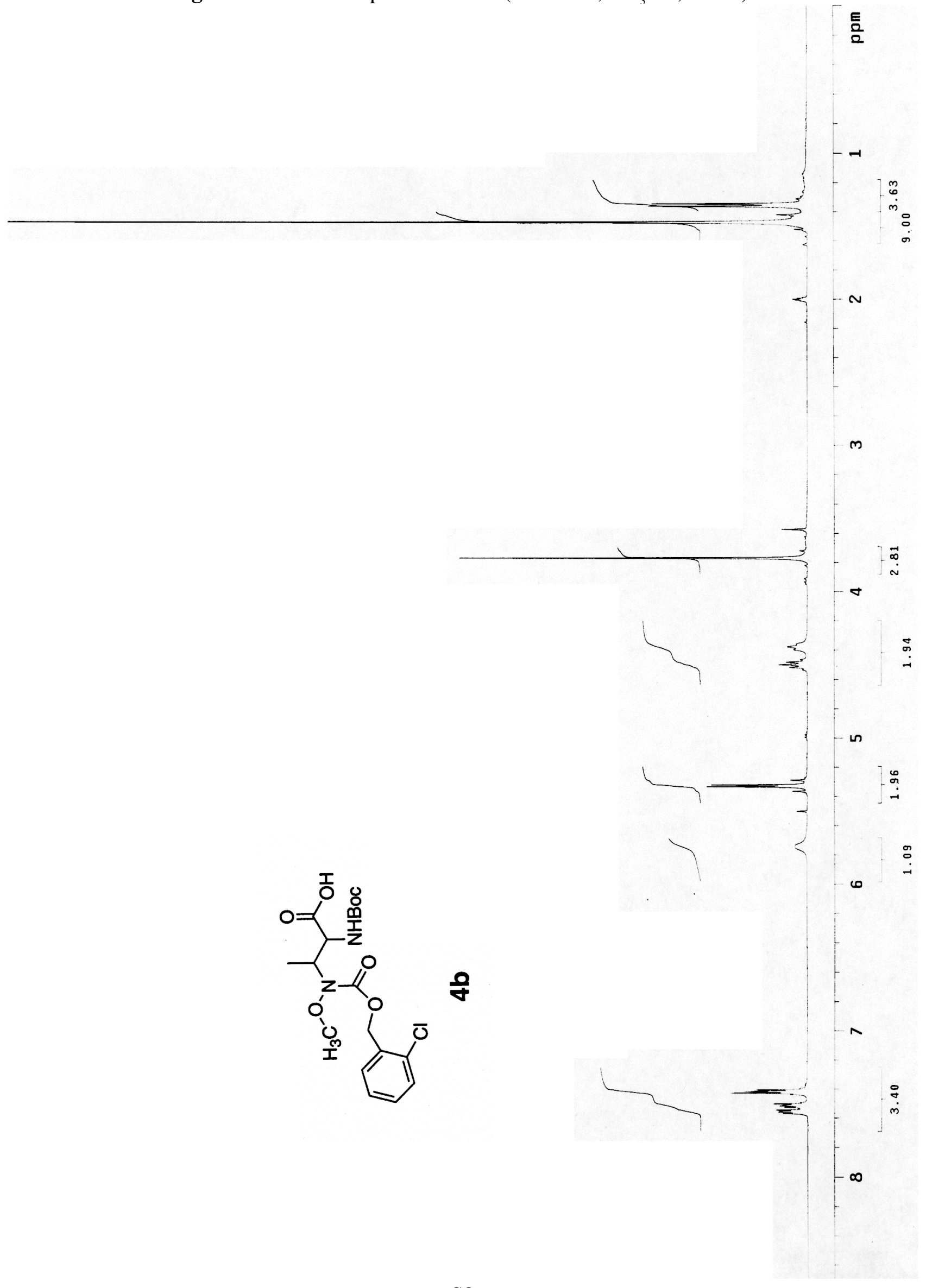


Figure S8. ${ }^{13} \mathrm{C}$ NMR spectrum of $\mathbf{4 b}\left(100 \mathrm{MHz}, \mathrm{CD}_{3} \mathrm{CN}\right)$.

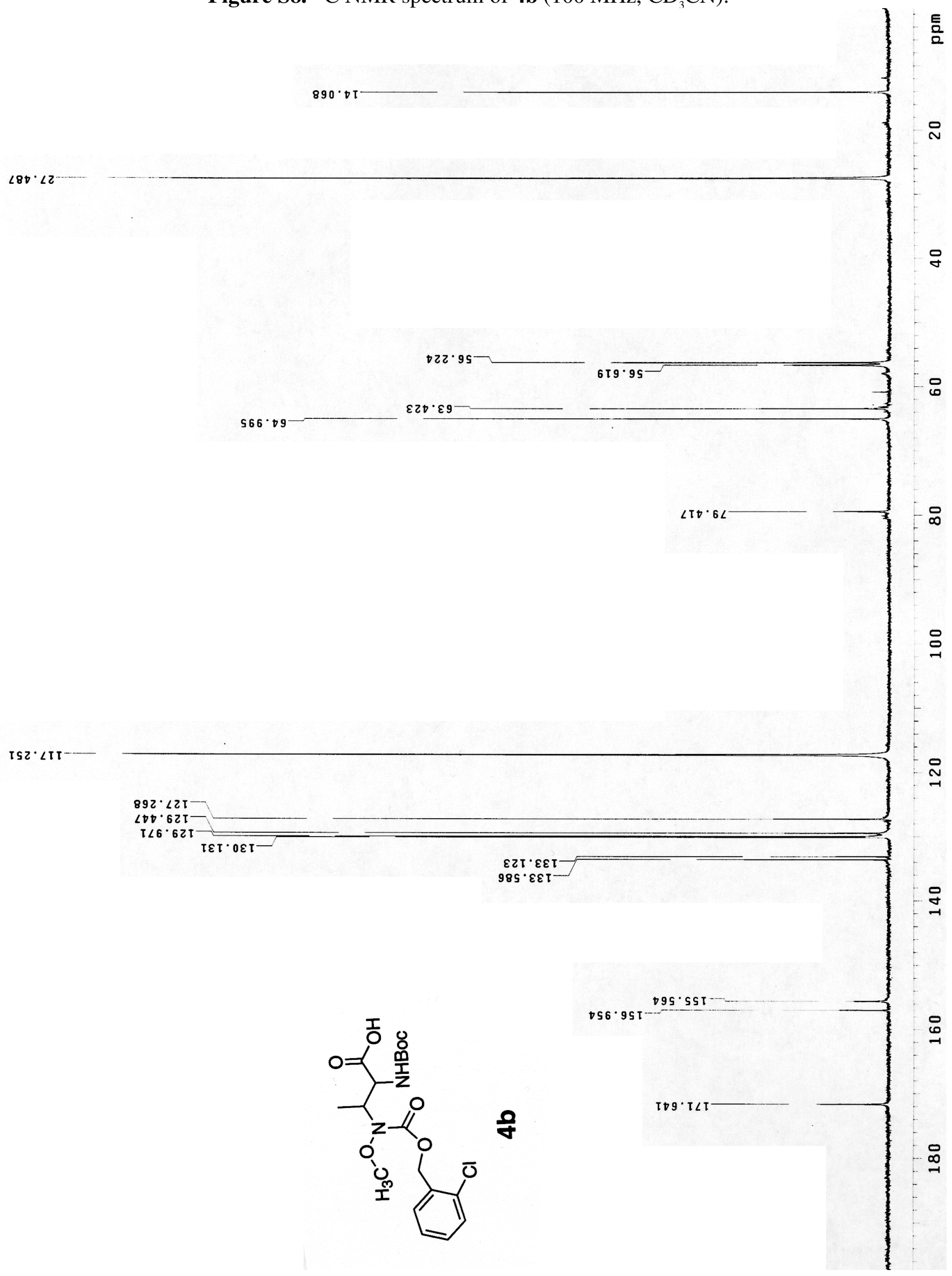


Figure S9. ${ }^{1} \mathrm{H}$ NMR spectrum of $5\left(400 \mathrm{MHz}, \mathrm{CDCl}_{3}\right)$.

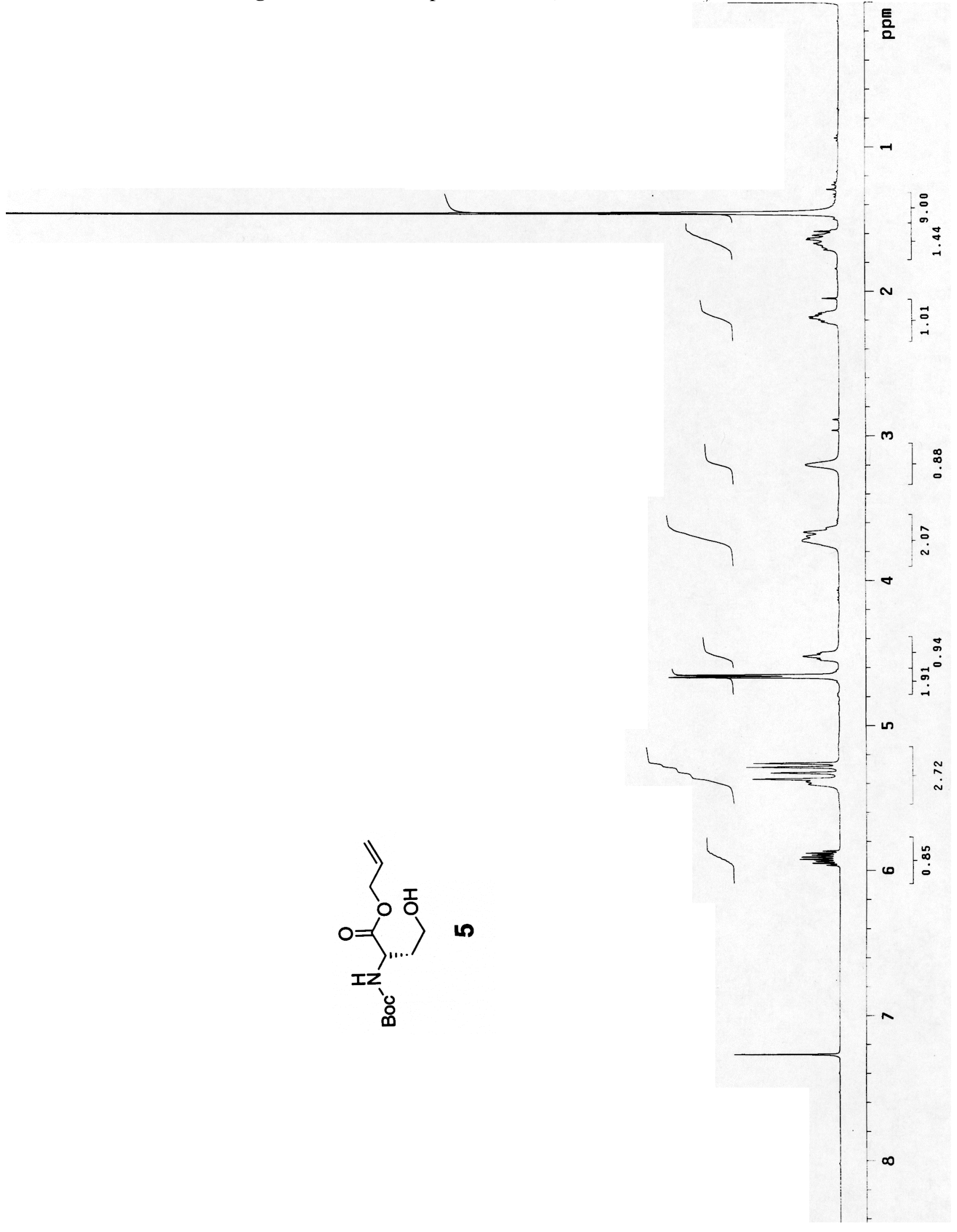


Figure S10. ${ }^{13} \mathrm{C}$ NMR spectrum of $5\left(100 \mathrm{MHz}, \mathrm{CDCl}_{3}\right)$.

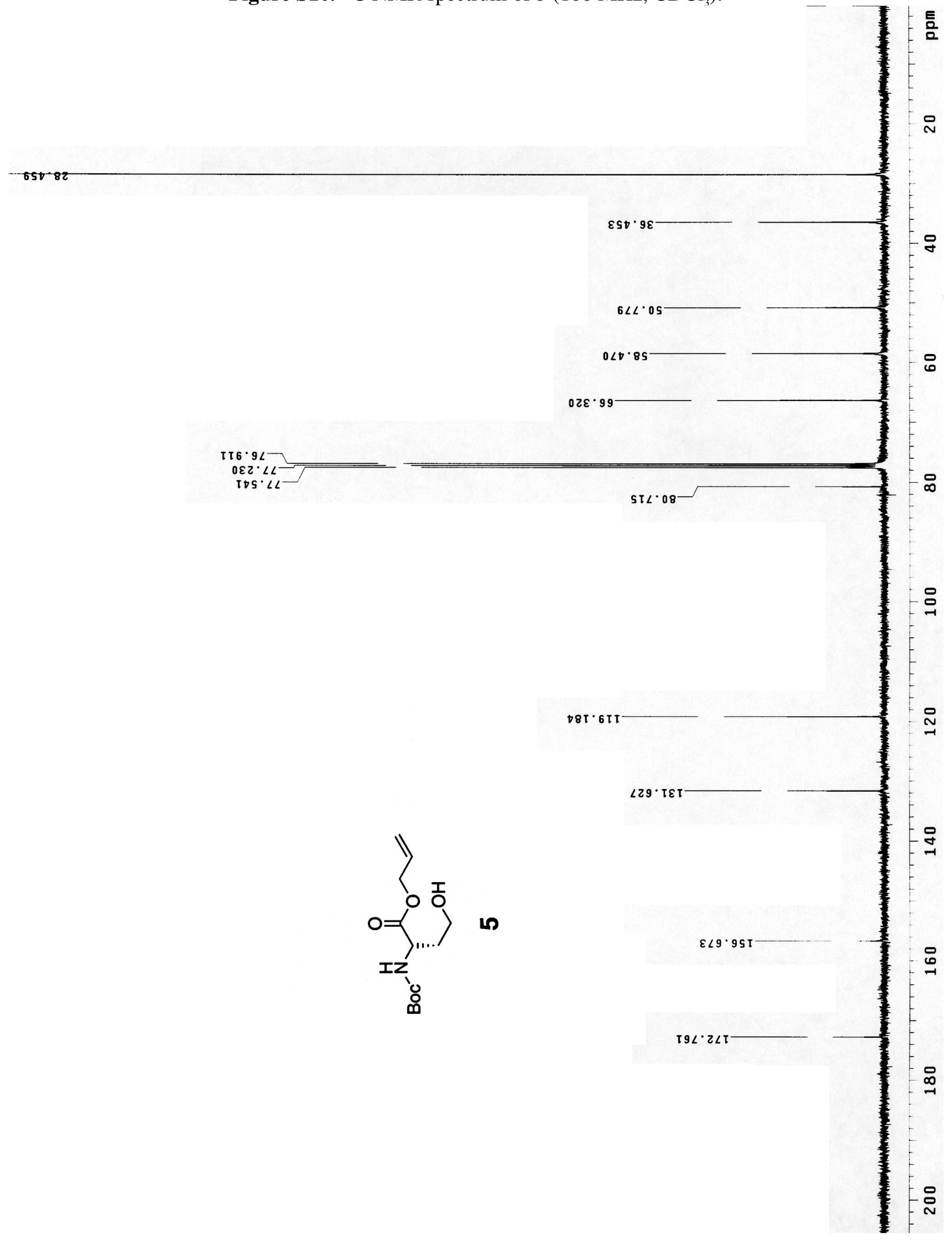


Figure S11. ${ }^{1} \mathrm{H}$ NMR spectrum of $6\left(400 \mathrm{MHz}, \mathrm{CDCl}_{3}\right)$.

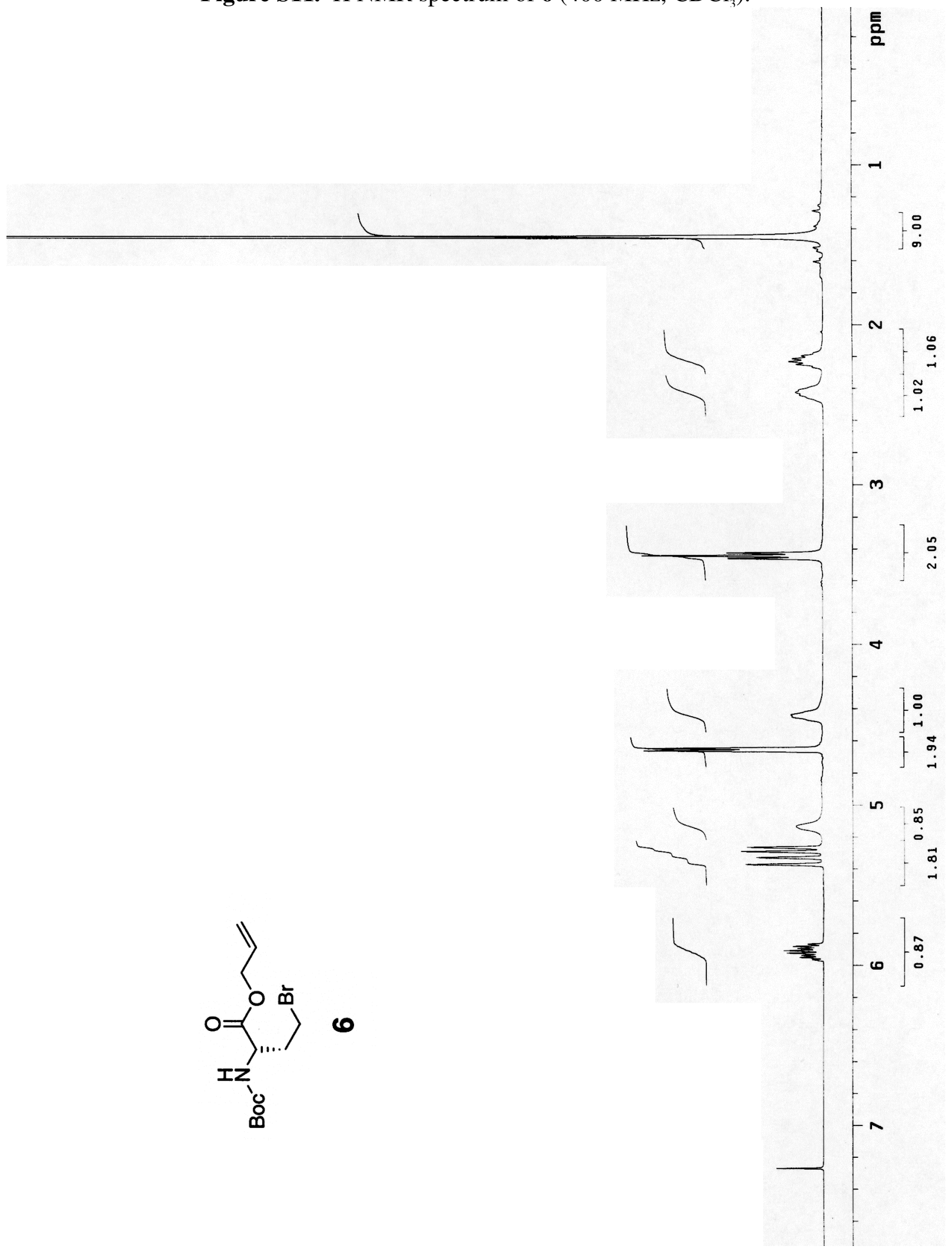


Figure S12. ${ }^{13} \mathrm{C}$ NMR spectrum of $6\left(100 \mathrm{MHz}, \mathrm{CDCl}_{3}\right)$.

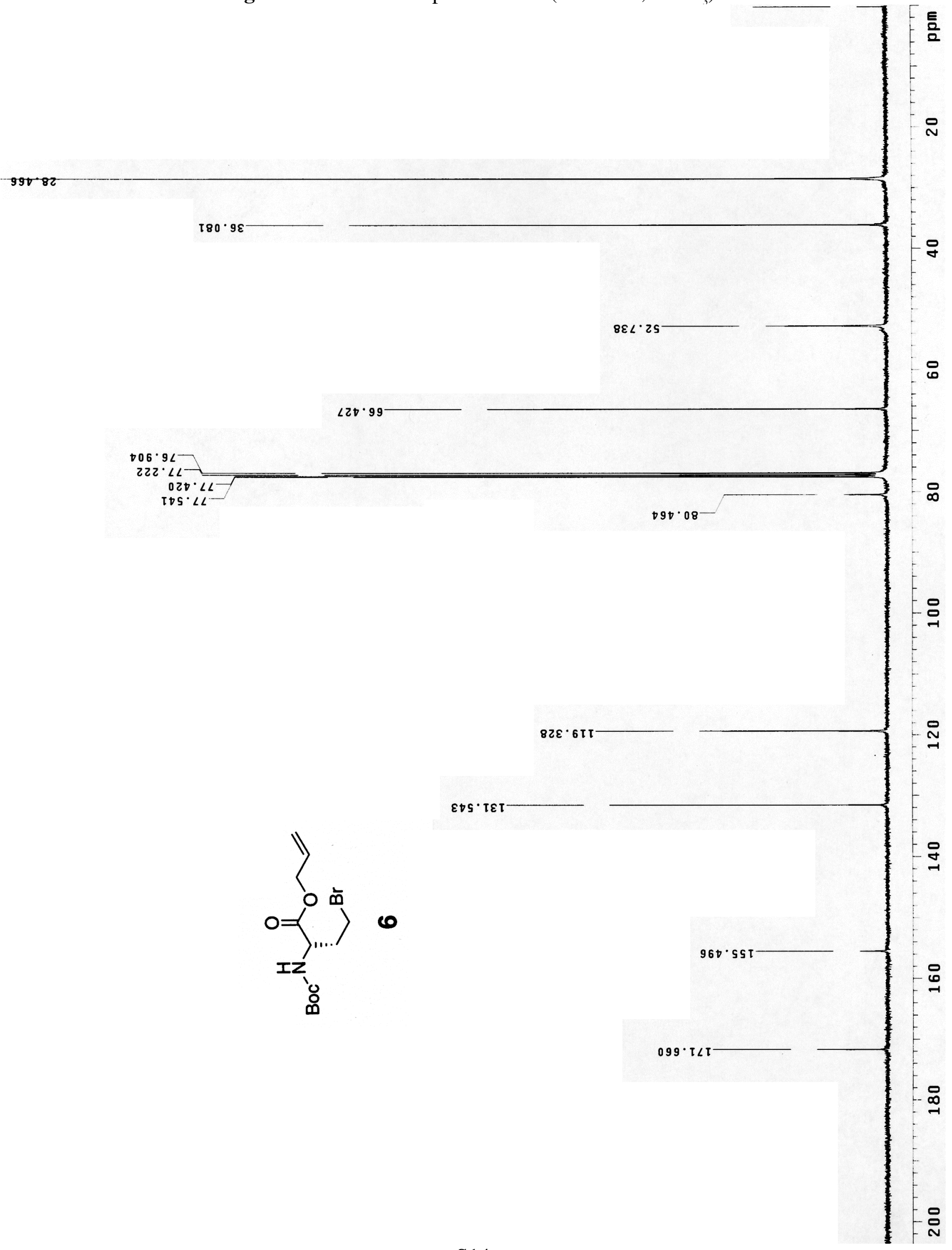


Figure S13. ${ }^{1} \mathrm{H}$ NMR spectrum of $7\left(400 \mathrm{MHz}, \mathrm{CDCl}_{3}\right)$.

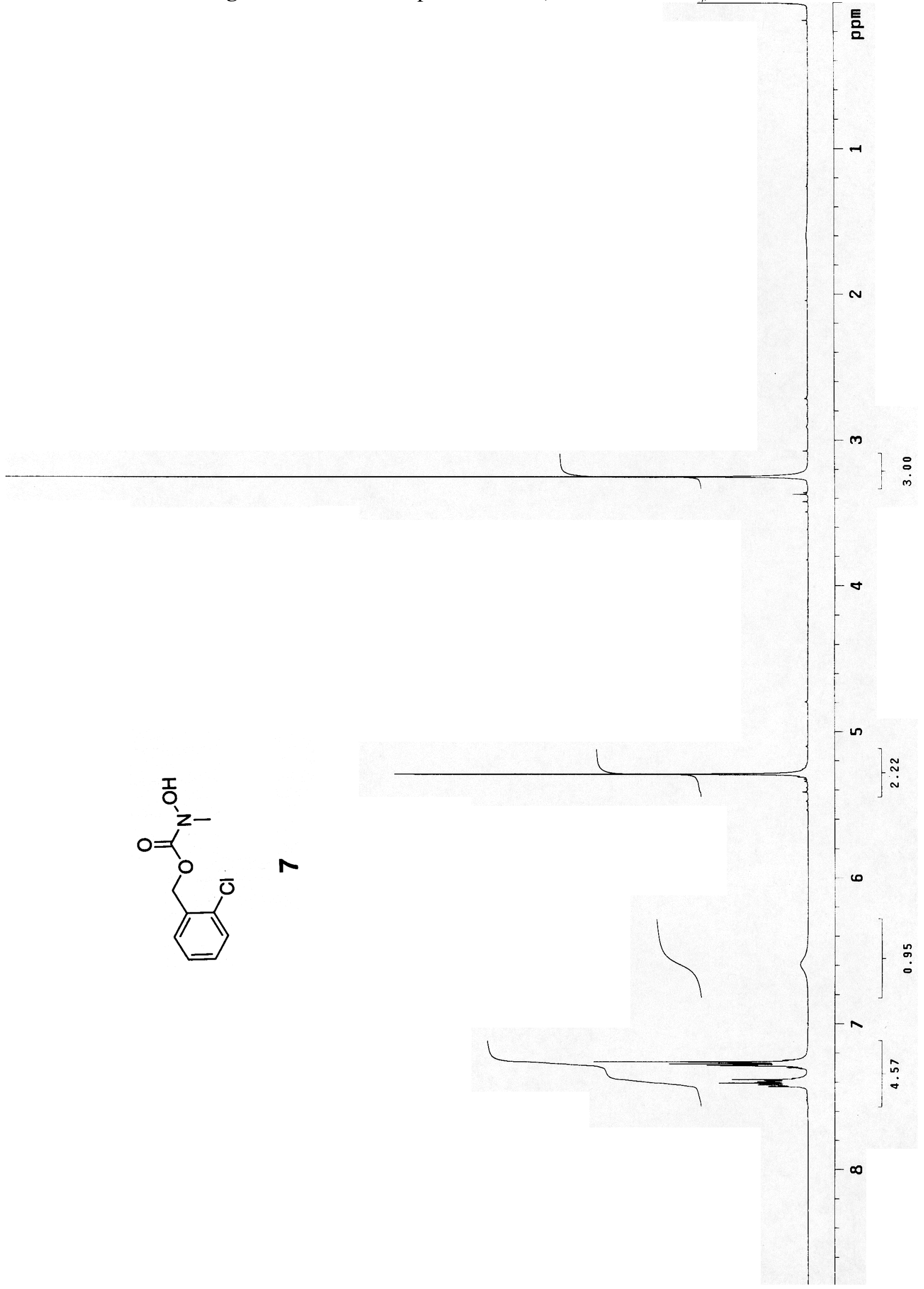


Figure S14. ${ }^{13} \mathrm{C}$ NMR spectrum of $7\left(100 \mathrm{MHz}, \mathrm{CDCl}_{3}\right)$.

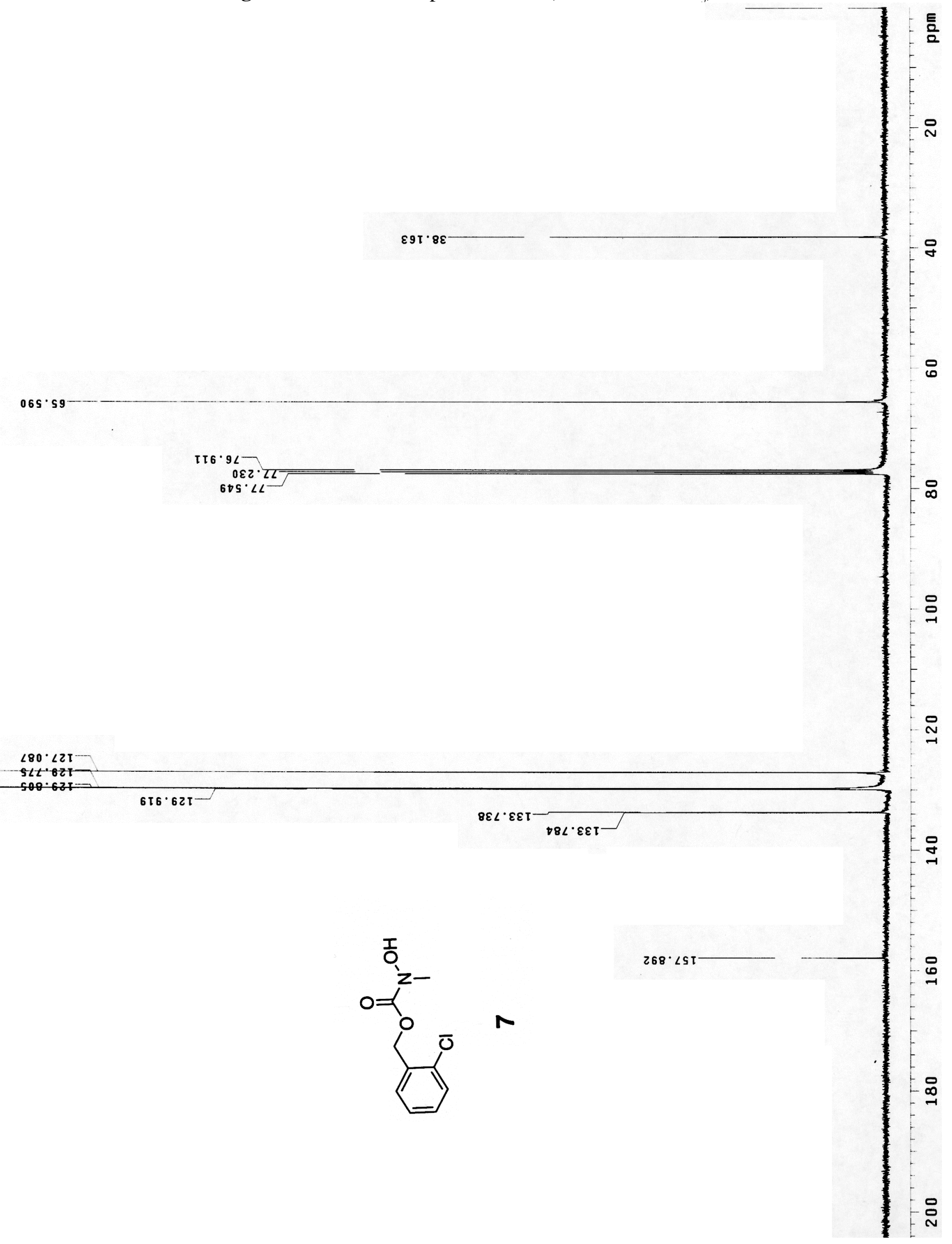


Figure S15. ${ }^{1} \mathrm{H}$ NMR spectrum of $8\left(400 \mathrm{MHz}, \mathrm{CDCl}_{3}\right)$.

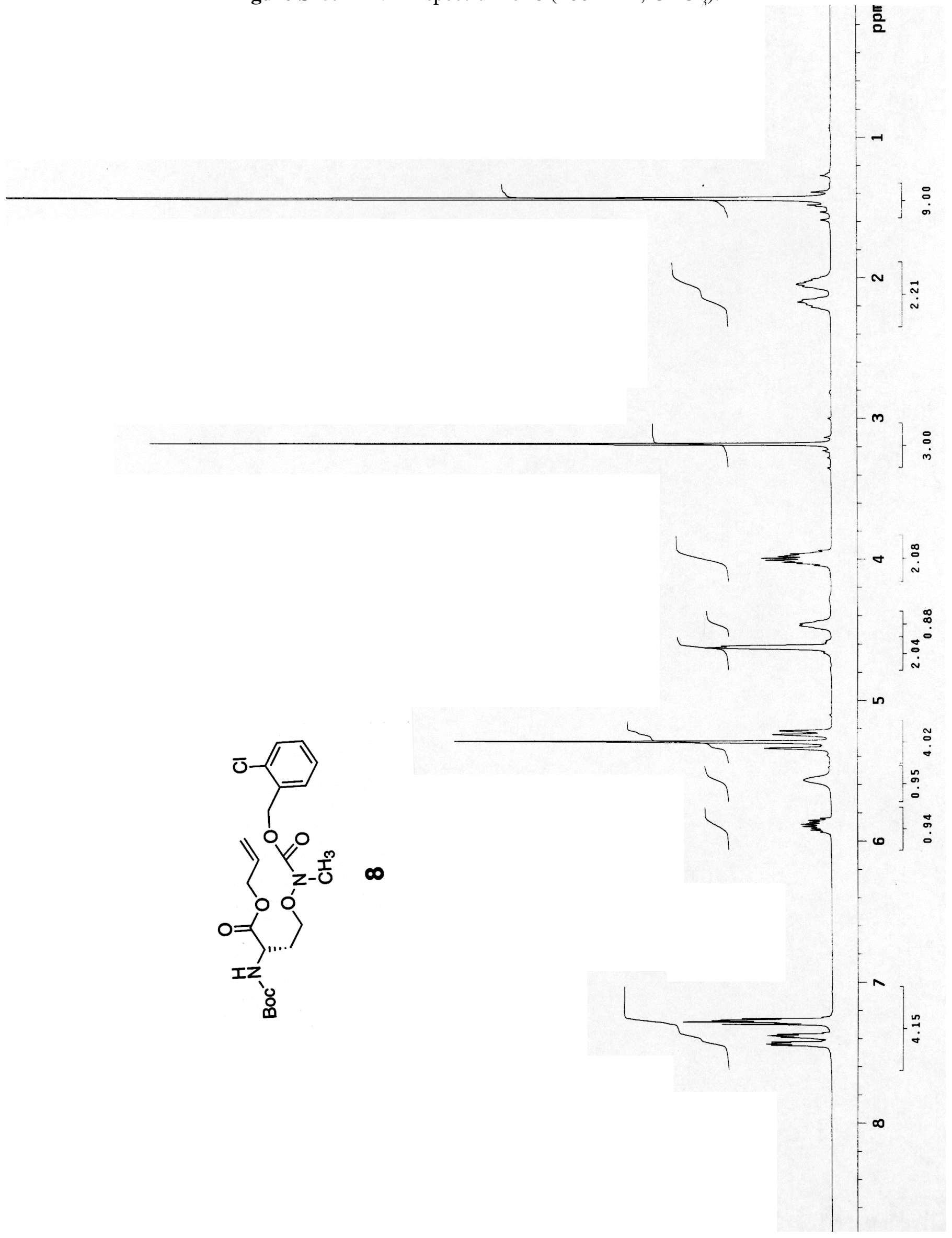


Figure S16. ${ }^{13} \mathrm{C}$ NMR spectrum of $8\left(100 \mathrm{MHz}, \mathrm{CDCl}_{3}\right)$.

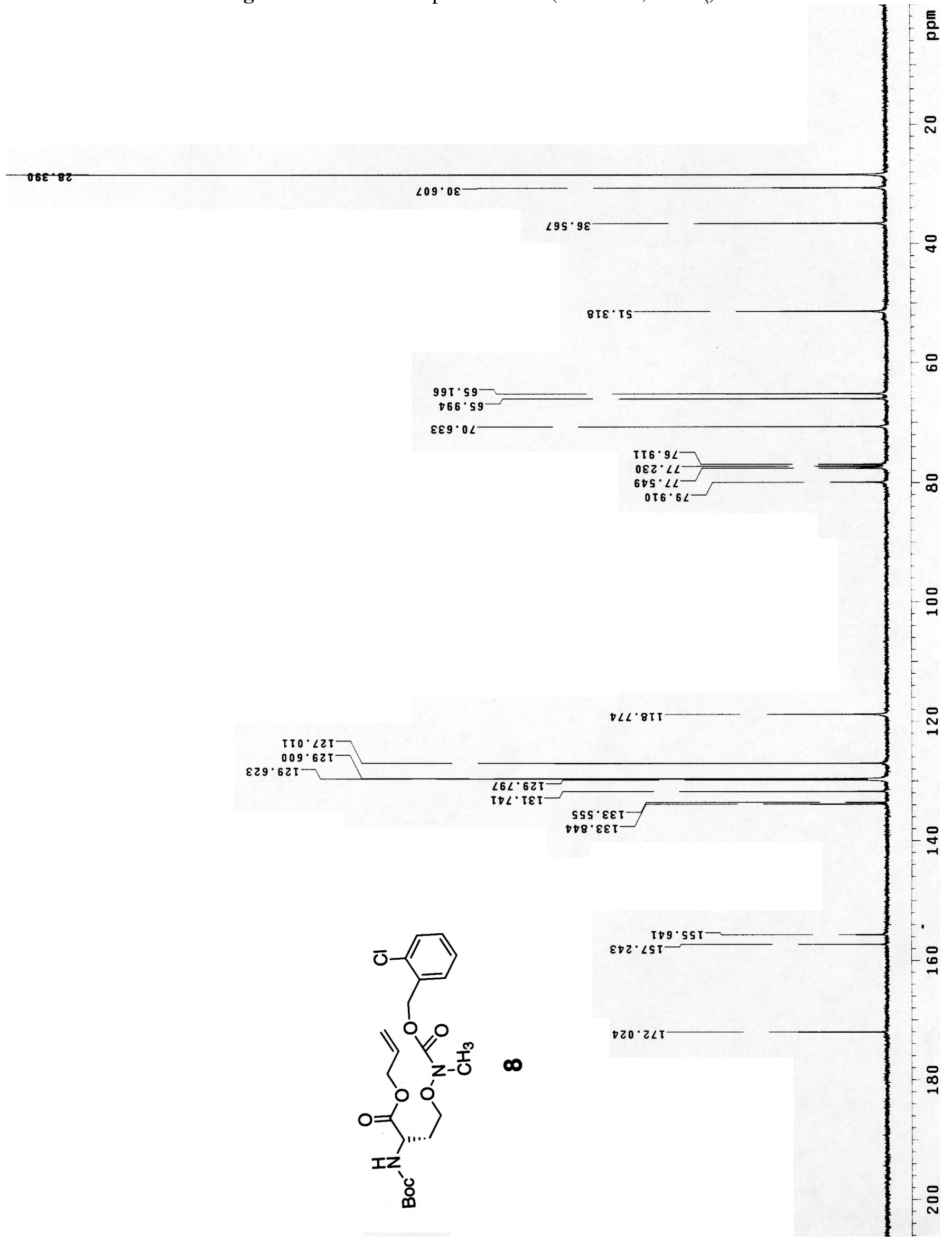


Figure S17. ${ }^{1} \mathrm{H}$ NMR spectrum of $9\left(400 \mathrm{MHz}, \mathrm{CDCl}_{3}\right)$.

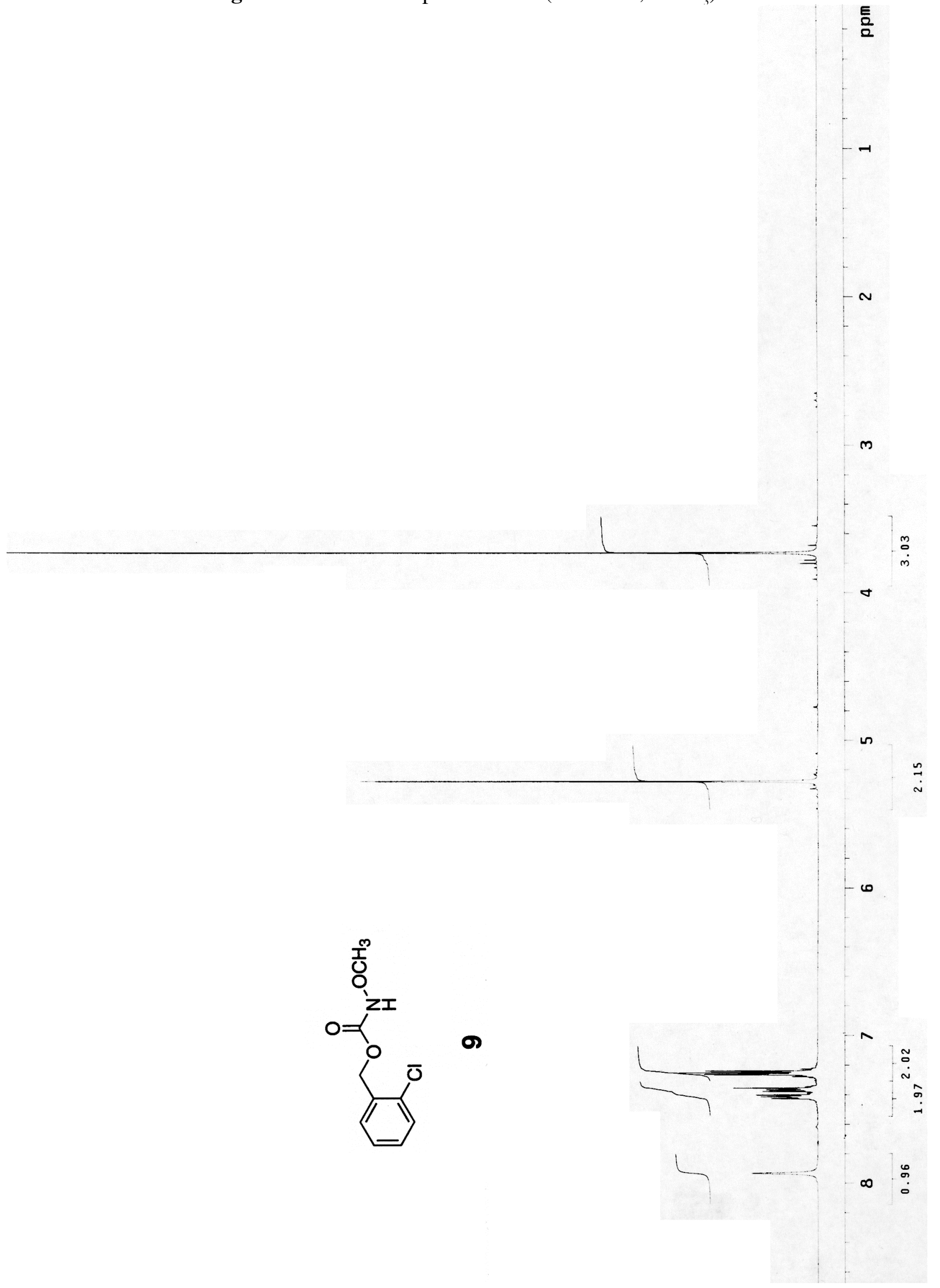


Figure S18. ${ }^{13} \mathrm{C}$ NMR spectrum of $9\left(100 \mathrm{MHz}, \mathrm{CDCl}_{3}\right)$.

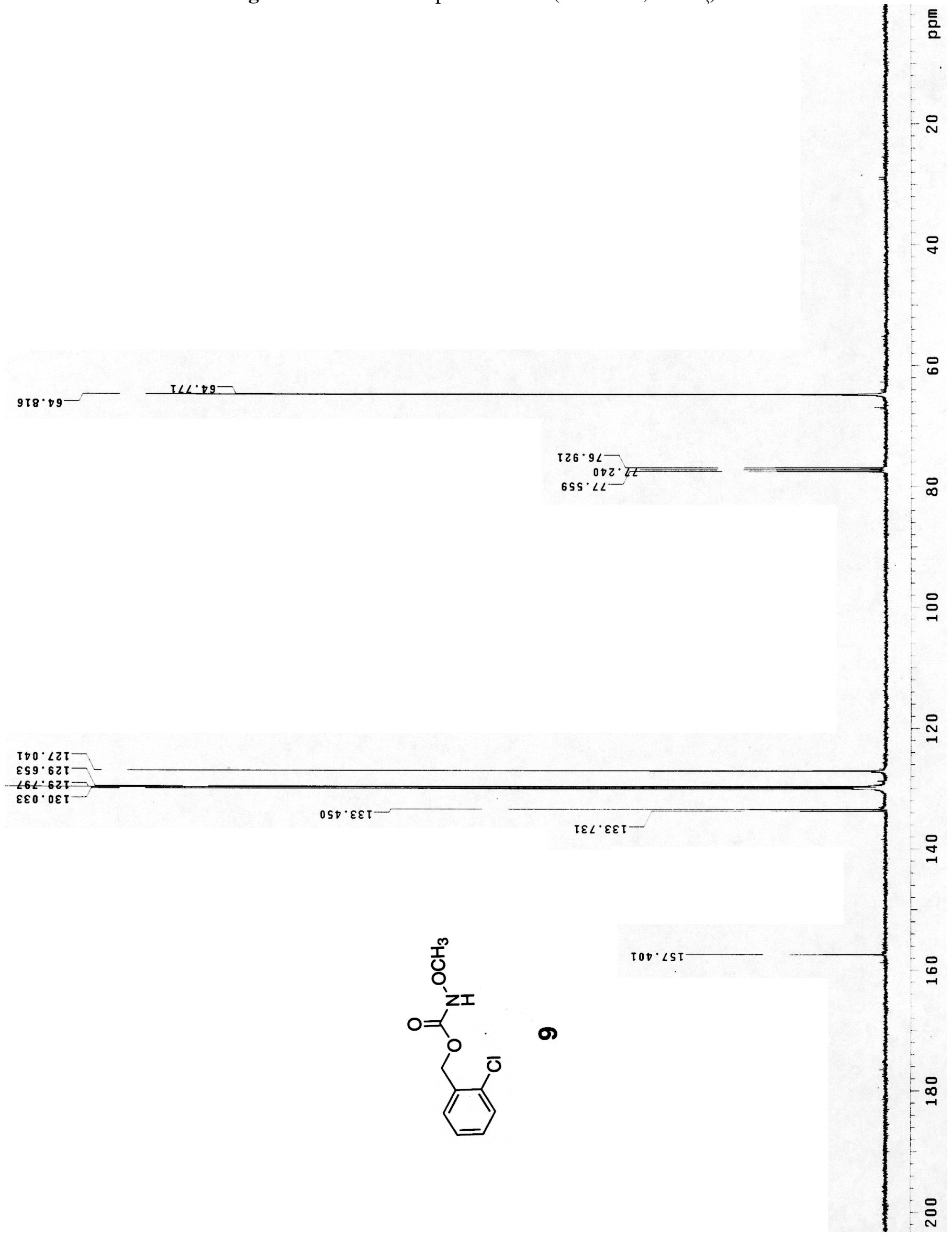


Figure S19. HPLC chromatograms of $\mathbf{1 4}$ and $\mathbf{1 8}$

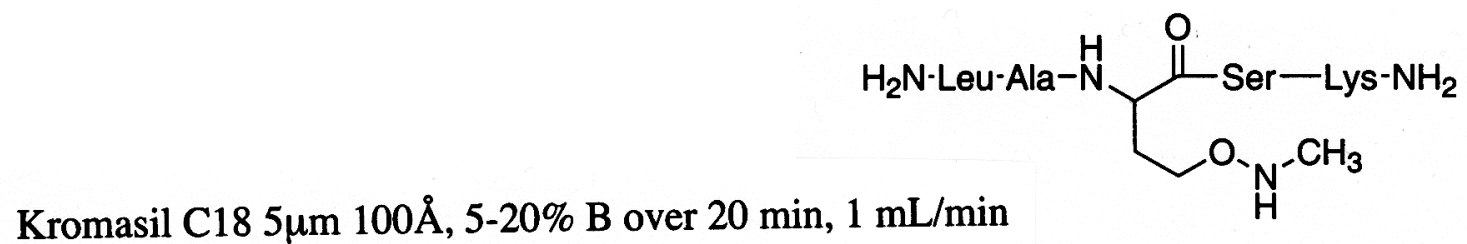

14

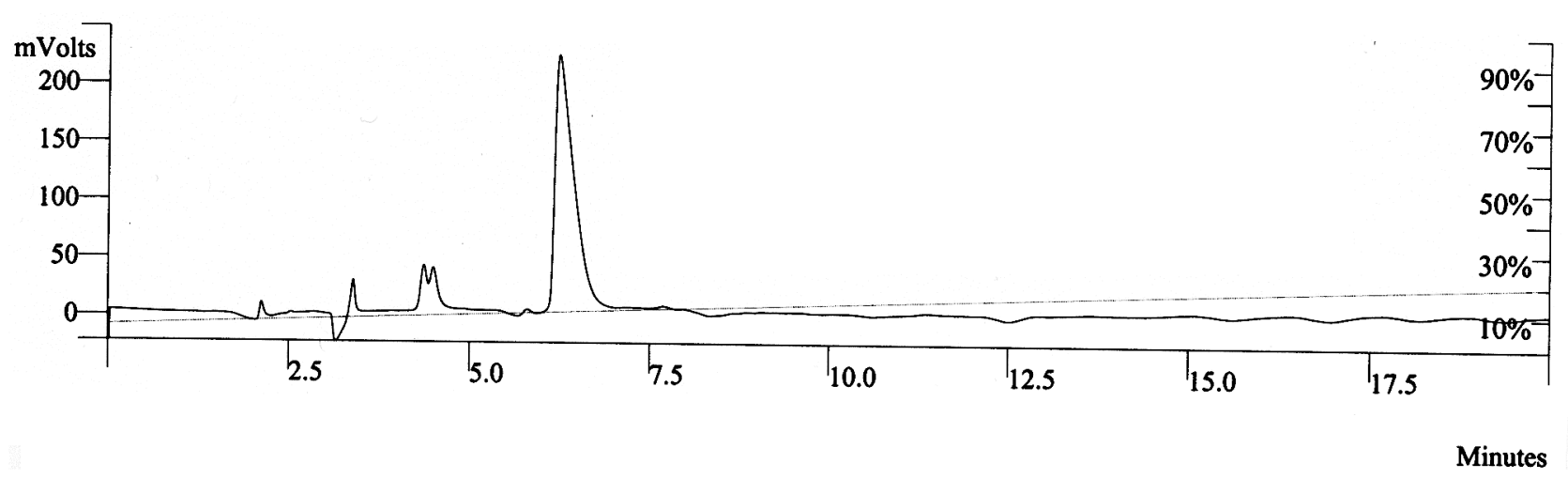

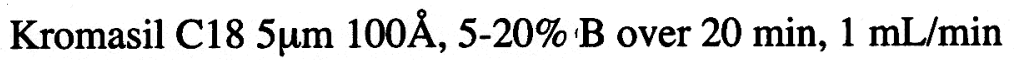

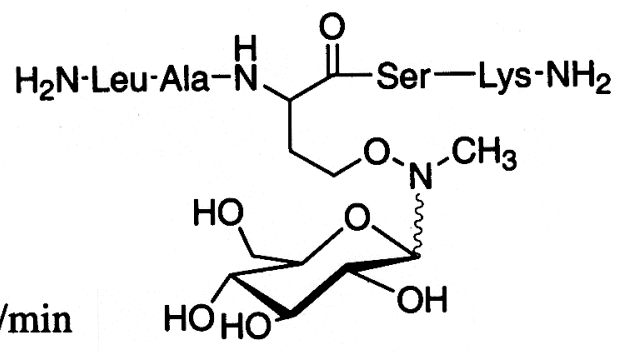

18

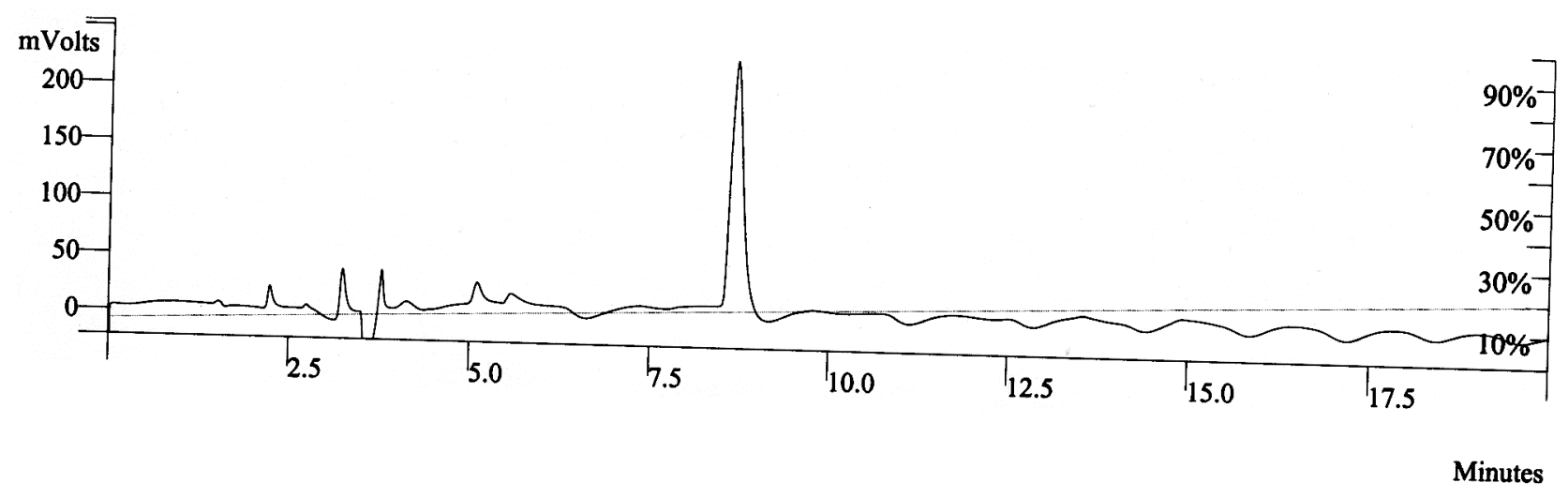


Figure S20. HPLC chromatograms of $\mathbf{1 5}$ and $\mathbf{1 9}$

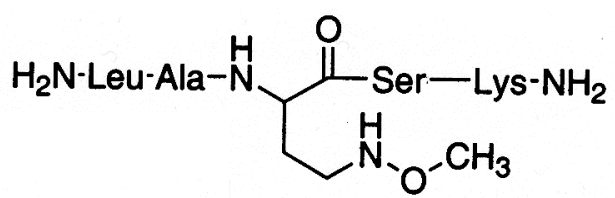

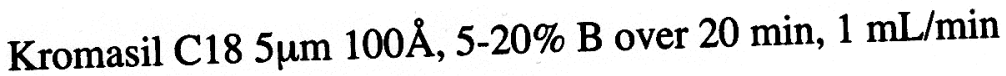

15

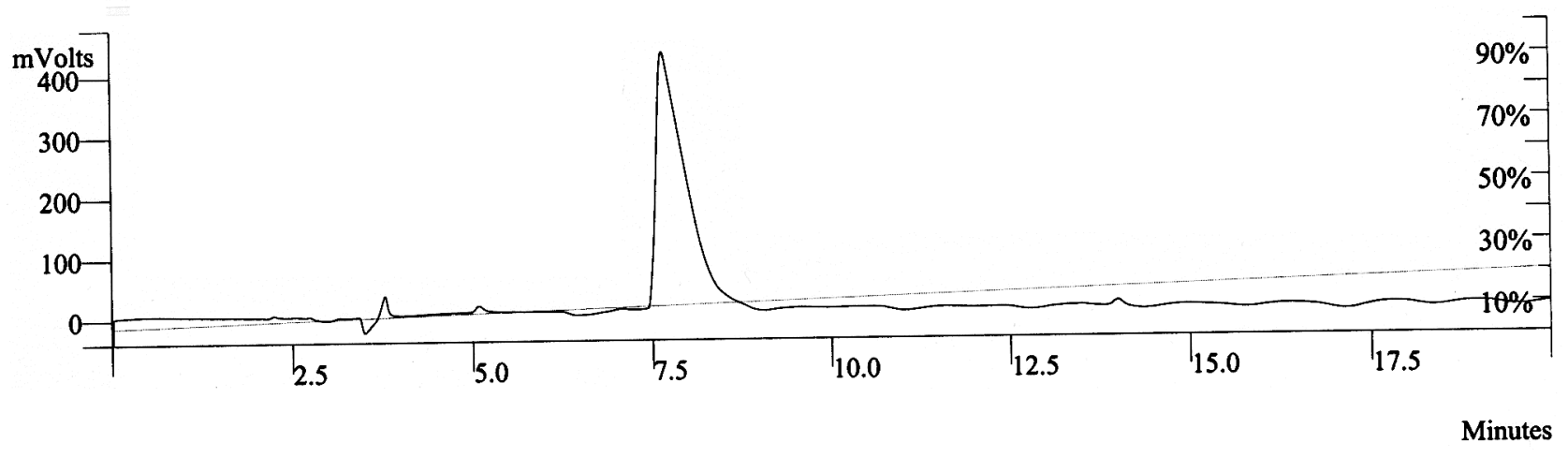

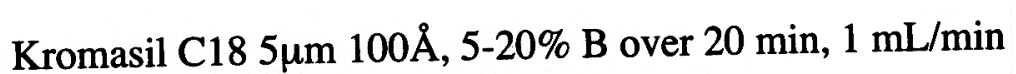

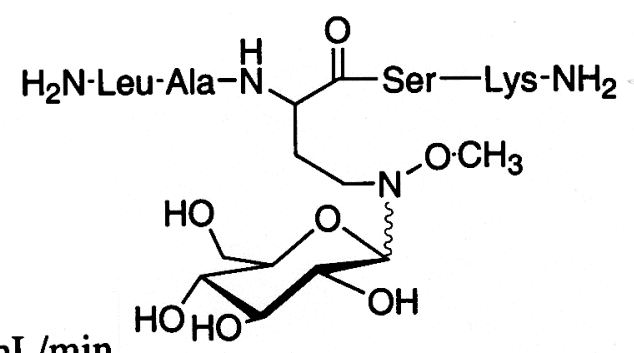

19

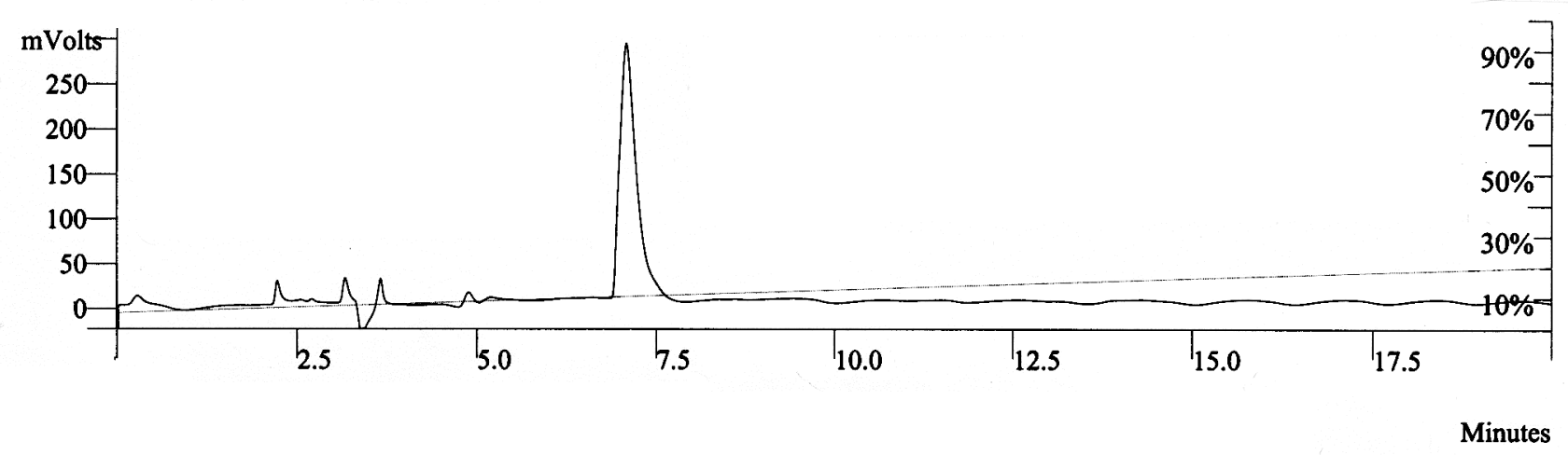


Figure S21. HPLC chromatograms of $\mathbf{1 6}$ and $\mathbf{2 0}$

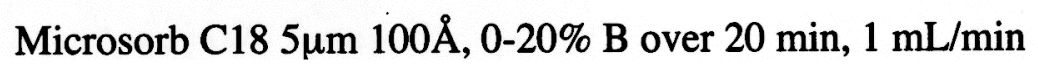<smiles>CONCC(N[14CH3])C(=O)[SeH2][AsH2]N</smiles>

16

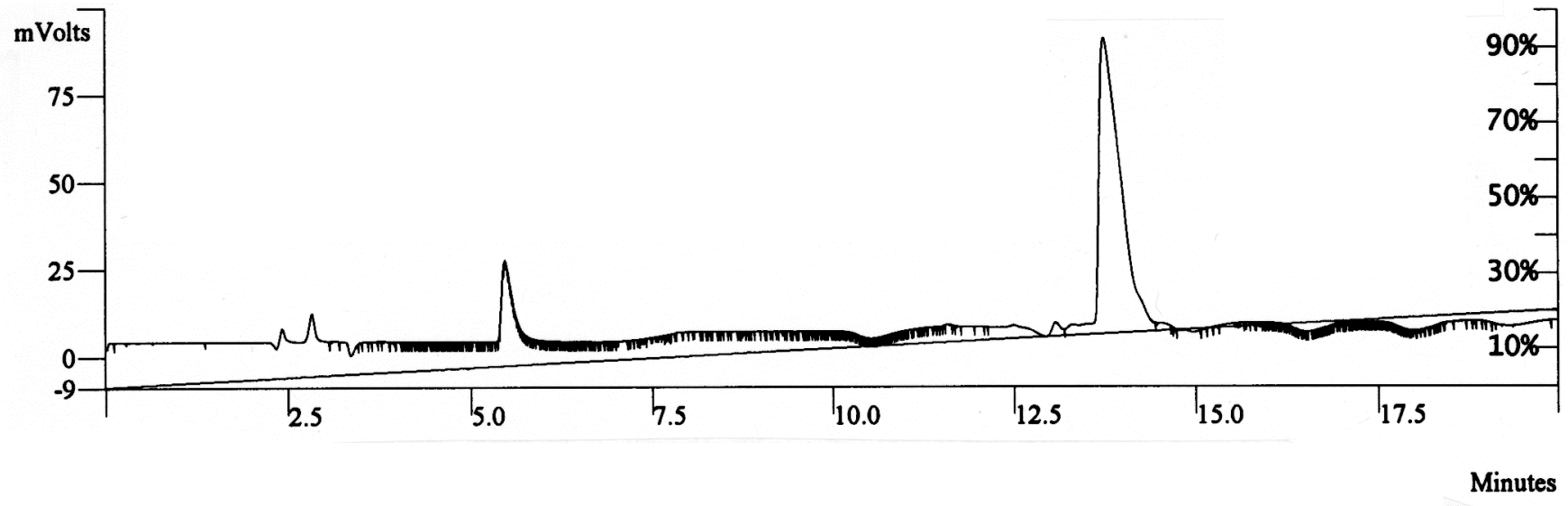

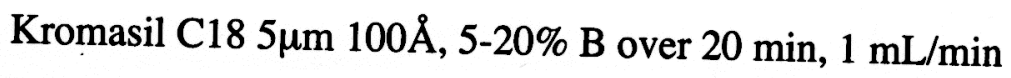

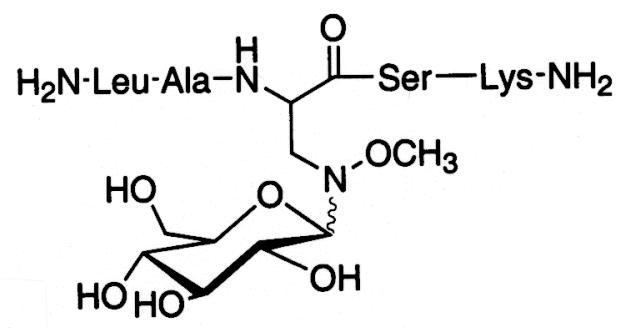

20

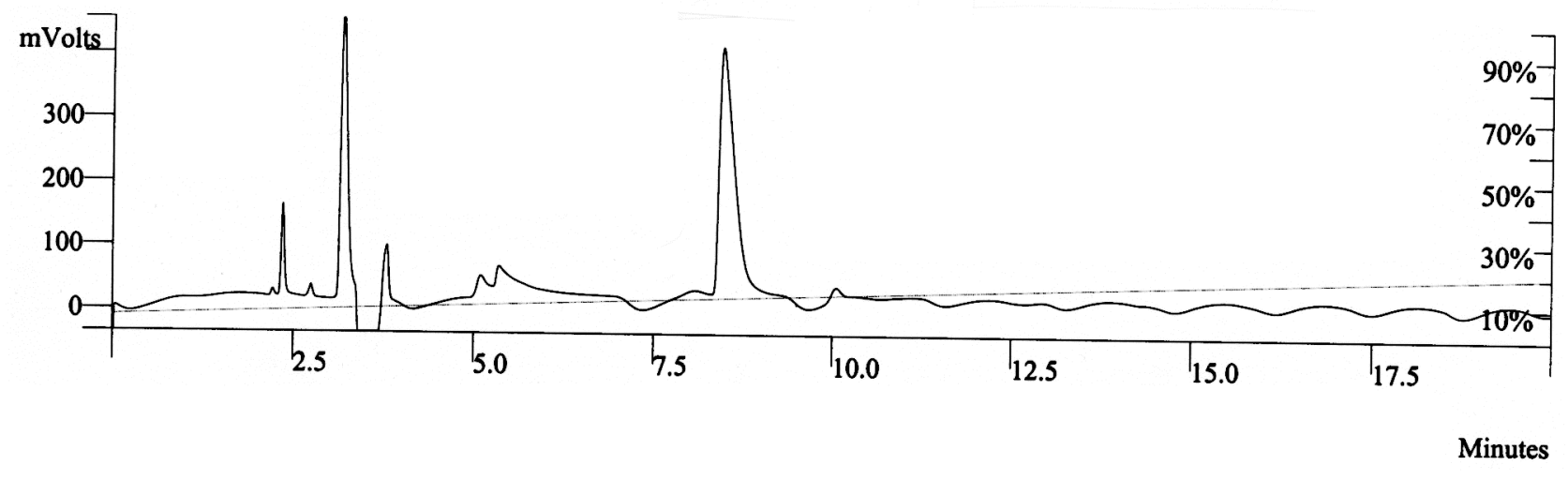


Figure S22. HPLC chromatogram of $\mathbf{1 7}$

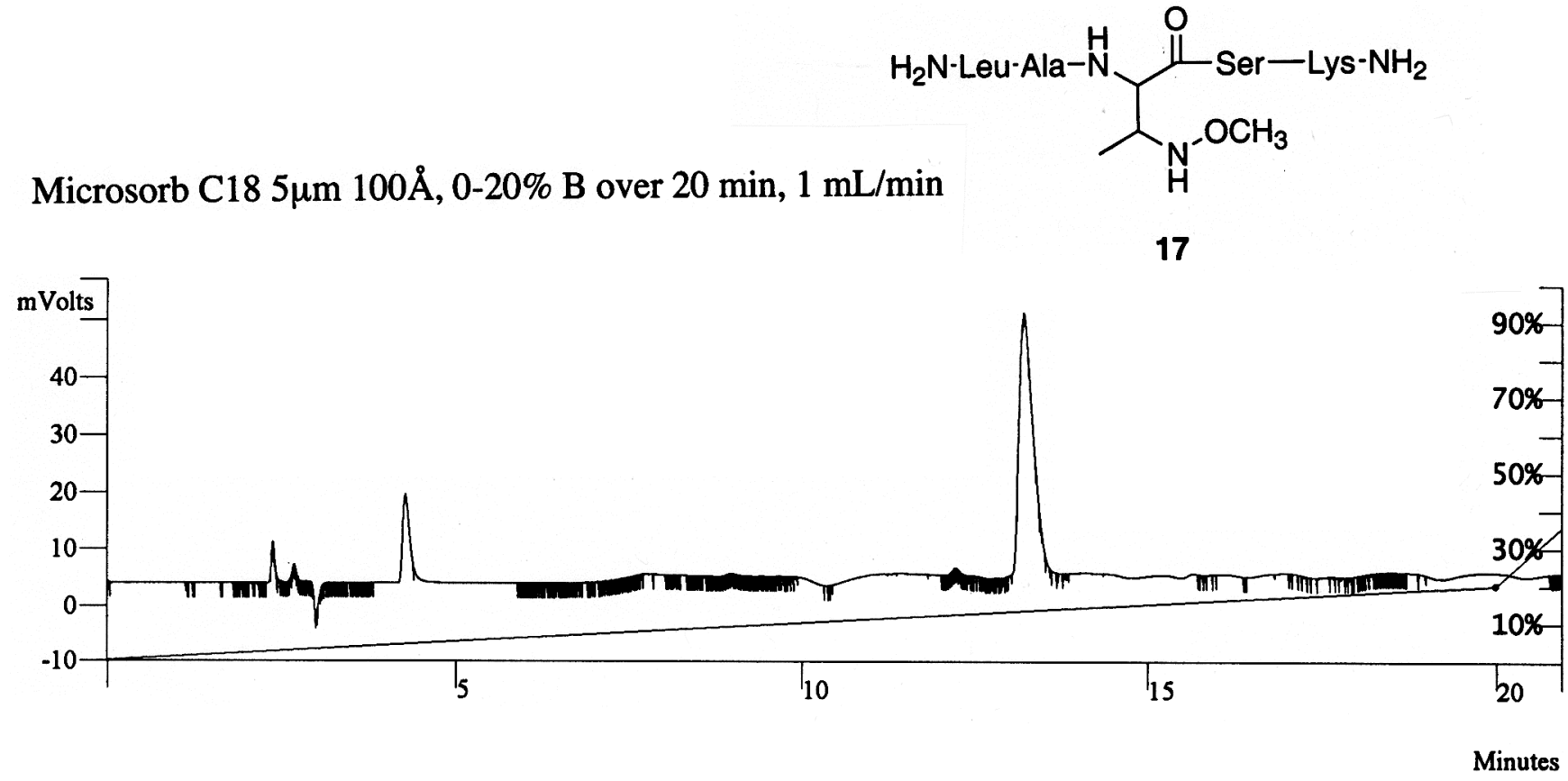

